\title{
A FURTHER CASE OF DOMINANCE INTERACTION IN MUS MUSCULUS
}

I. R. GOODWINS

Department of Genetics, Cambridge University

Received 24.iii.57

IT was reported by Snell (I93I) that in the presence of homozygous non-agouti $(a)$ and pink-eyed dilution $(p)$, the heterozygous condition black-brown $(B b)$ is distinguishable, as a lighter colour, from the homozygous black $(B B)$. Wallace (1953) pointed out that this constitutes a reduction of the dominance of $B$ by the $p$ locus, and demonstrated that the converse is also true, namely that the $b$ locus reduces the dominance of $P$. Thus the nine genotypes available at these two loci comprise six distinct phenotypes (table I).

TABLE :

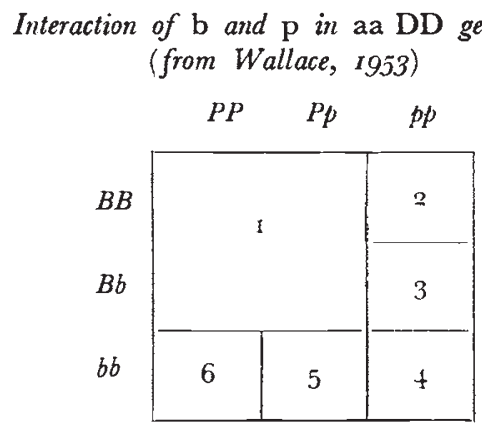

In a stock involving these same factors, in which $P P$ was backcrossed to $P p$, and in which Maltese dilution $(d)$ was also segregating, Wallace has also noticed two colour phenotypes in $B b d d$ and $b b d d$, namely dark and pale black dilutes, and dark and pale brown dilutes. On her suggestion that the "darks" in each case are $P P$ and the "pales" $P p$, I have tested for the presence of $p$, several "darks" and "pales" among both the black and the brown clilutes from the backcross. In fourteen cases out of seventeen, the $p$ genotype conformed to expectation. Since for each animal tested there was an equal chance of a right and a wrong classification, a simple $\chi^{2}$ testing the equality of the number of right and wrong diagnoses gives the probability of obtaining this result by chance. $\chi^{2}$ is $7 \cdot I I 76$ for $I$ d.f., probability $<0 \cdot 01$. Thus it may safely be concluded that, in the presence of $d d$, further genotypes at the $b$ and $p$ loci are distinct. These interactions may be summarised as follows (table 2).

The remaining phenotypic distinctions possible in $B$ and $P$ are being investigated in this Department, and it is hoped that a full table of interactions in $d d$ genotypes may be published shortly as a 
counterpart to that for $D D$ genotypes. It should perhaps be pointed out that in all cases non-agouti mice are being used. It is possible to make many of the distinctions on agouti mice, if the non-yellow part of the fur is examined. Thus Snell's insistence on $a a$ is not absolutely necessary, but it is helpful.

In order to confirm the present result and to examine the possible: effect of minor colour factors, the genotypes I to 4 of table 2 were each reproduced from matings not segregating at the $p$ locus, i.e. from matings whose progeny could be classificd with complete certainty. These were :

\section{(i) $P P B b$ a $a d d \times p p b b$ a $a d d$ \\ (ii) $P P B b$ a a $d d \times P P$ bb an $d d$.}

Five mice of each genotype were skinned at approximately 28 days old, making twenty skins in all. These were displayed, each set of

TABLE 2

Interaction of $\mathrm{b}$ and $\mathrm{p}$ in aa $\mathrm{dd}$ genolypes

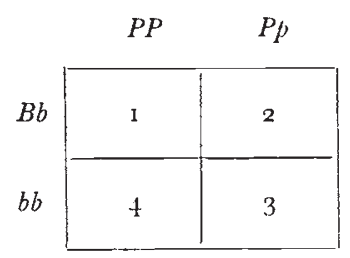

five separately, and the skins in each set were numbered I to $\bar{j}$. Fifteen people in the Department who were not associated with the experiment were asked independently to say which were the pale and which the dark sets of skins. Among both the blacks and the browns, their classification, in all cases, coincided with the $P p$ and $P P$ classification. Thus the effect of the $P / P$ gene substitution is indisputable.

Each person was then asked to arrange the skins within each set in order of increasing intensity. From the tabulation of these orders, it was found that in none of the sets was there unanimous agreement upon any order, but in all sets one or two of the skins were classified by the majority as definitely paler, or darker, than the rest. Thus it is clear that slight variation exists within each phenotype : this nay be due to the segregation of minor colour factors or to environmental differences such as age and its effect on the stage of moult. The median skin, that is, the one occurring most frequently as number 3 in the tabulation of orders, was selected to demonstrate the contrasts between the four phenotypes and can be seen in this Department.

It is conceivable that minor genetic and environmental fluctuations could, in a replication of this investigation, produce slightly different degrees of contrast between the four phenotypes-and in fact some overlapping may be possible. Short of complete standardisation of 
residual genotype and environment, a laborious task, the exact interrelations cannot be established. However, such a replication, if it confirmed the contrasts so far observed, would enable the less clear distinctions such as may exist between the $B B$ and $B b$ genotypes and the $P P$ and $P p$ ones, to be made with confidence. For this reason, the fuller investigation now being carried out, includes a replication of the present work.

To summarise : Wallace's work demonstrates the power of homozygous $b$ to reduce the dominance of $P$ over $p$. The present work shows that, with the help of a third factor diluting the wild black colour, namely $d$, the effect of $b$ is even stronger, this dominance being incomplete in heterozygous as well as homozygous $b$ genotypes. There is some variation within each phenotype, which may be due to minor genetic factors or to environmental causes.

\section{REFERENCES}

SNELL, G. D. I931. Inheritance in the house mouse. Genetics, I6, 42.

WALLACE, M. E. 1953. A case of mutual reduction of dominance observed in Mus musculus. Heredity, 7, 435 . 